Article

\title{
Computing the Scale of an Endomorphism of a totally Disconnected Locally Compact Group
}

\author{
George A. Willis \\ School of Mathematical and Physical Sciences, University of Newcastle, University Drive, \\ Callaghan NSW 2308, Australia; george.willis@newcastle.edu.au; Tel.: +61-2-4921-5666; Fax: +61-2-4921-6898
}

Received: 28 August 2017; Accepted: 9 October 2017; Published: 20 October 2017

\begin{abstract}
The scale of an endomorphism of a totally disconnected, locally compact group $G$ is defined and an example is presented which shows that the scale function is not always continuous with respect to the Braconnier topology on the automorphism group of $G$. Methods for computing the scale, which is a positive integer, are surveyed and illustrated by applying them in diverse cases, including when $G$ is compact; an automorphism group of a tree; Neretin's group of almost automorphisms of a tree; and a $p$-adic Lie group. The information required to compute the scale is reviewed from the perspective of the, as yet incomplete, general theory of totally disconnected, locally compact groups.
\end{abstract}

Keywords: locally compact group; endomorphism; scale; tree; Neretin's group; Thompson's group; $p$-adic Lie group

\section{Introduction}

Let $G$ be a totally disconnected, locally compact (t.d.l.c.) group. A fundamental theorem about t.d.1.c. groups, proved by van Dantzig in the 1930s, see [1] and ([2] Theorem II.7.7), asserts that $G$ has a base of neighbourhoods of the identity consisting of compact open subgroups. These subgroups are important for the definition of the scale of endomorphisms $\alpha: G \rightarrow G$, which is a positive integer gauging the action of $\alpha$. The precise definition is given in Section 2. Computing the scale is a problem which, as will be seen in examples, depends very much on a description of the group $G$ and of the endomorphism $\alpha$ (the examples in fact treat inner automorphisms), and one way to evaluate our understanding of general t.d.l.c. groups is whether we can carry out this computation.

Sections 3 and 4 describe two approaches to computing the scale and use them in examples. The first approach is the 'spectral radius formula' given by Rognvaldur Möller in [3], and the second uses the structure theorem given in $[4,5]$ for compact open subgroups at which the scale is attained. One of the examples in Section 4 is a $p$-adic Lie group and it is seen that the scale may be computed in terms of eigenvalues in the Lie algebra and minimising subgroups in terms of eigenvectors. This observation, together with the spectral radius formula, results about the scale such as in [6], and applications of scale techniques to answer questions about t.d.l.c. groups which are answered in the connected case with approximation by Lie group methods $[7,8]$, indicate that scale techniques may substitute for Lie methods in some circumstances. Motivation for developing improved methods for computing the scale is provided by these considerations.

Throughout the paper, $\operatorname{End}(G)$ will denote the monoid of continuous endomorphisms of $G$; $\operatorname{Aut}(G)$ will be the group of automorphisms of $G$; and $\operatorname{Inn}(G)$ the group of inner automorphisms of $G$.

\section{The Scale of an Endomorphism}

The scale was defined first for inner automorphisms of a t.d.l.c. group in $[4,5]$ and the definition was extended to endomorphisms in [9]. For this definition, note that, if $V$ is a compact open subgroup of $G$ and $\alpha$ a continuous endomorphism, then $\alpha(V) \cap V$ is an open subgroup of the compact group $\alpha(V)$ and hence the index $[\alpha(V): V \cap \alpha(V)]$ is finite. 
Definition 1. Let $\alpha \in \operatorname{End}(G)$. The scale of $\alpha$ is the positive integer

$$
s(\alpha):=\min \{[\alpha(V): V \cap \alpha(V)] \mid V \leq \text { G compact and open }\}
$$

Any $V$ at which the minimum is attained is minimising for $\alpha$.

The scale and related concepts have been used in papers such as $[7,8,10]$ to answer questions concerning t.d.l.c. groups. Many applications apply results to the scale function on $G$ induced by the conjugation map $G \rightarrow \operatorname{Inn}(G)$. For this scale function, we have the following, which is proved in [4].

Theorem 1. The scale function $s: G \rightarrow \mathbb{N}$ is continuous for the group topology on $G$ and the discrete topology on $\mathbb{N}$.

This theorem is used in [10] to answer a question of K. H. Hofmann about the structure of t.d.l.c. groups. Ideas from the proof of the theorem have also been used to answer questions about the Chabauty space of closed subgroups of a t.d.l.c. group in [11,12].

It is a natural question whether the scale function is continuous with respect to the group topology with which $\operatorname{Aut}(G)$ is usually equipped, namely, the Braconnier topology, see [13]. That is not the case, however, as may be seen as follows.

Example 1. Let $G=\left(\mathbb{K}((t))^{2},+\right)$ be the additive group of the 2-dimensional vector space over the field $\mathbb{K}((t))$ of formal Laurent series over the finite field $\mathbb{K}$ and let $\alpha$ be the automorphism $\alpha(f, g)=\left(t f, t^{-1} g\right)$. Then, $s(\alpha)=|\mathbb{K}|$. Define, for each $n \in \mathbb{Z}, \alpha_{n}(f, g)=\left(f^{(n)}, g^{(n)}\right)$ where

$$
f^{(n)}=\sum_{k=-\infty}^{n} f_{k-1} t^{k}+\sum_{k=n+1}^{\infty} f_{k} t^{k} \quad \text { and } \quad g^{(n)}=\sum_{k=-\infty}^{n-1} g_{k+1} t^{k}+f_{n} t^{n}+\sum_{k=n+1}^{\infty} g_{k} t^{k} .
$$

Then, it may be verified that $\alpha_{n} \rightarrow \alpha$ in the Braconnier topology as $n \rightarrow \infty$ but $s\left(\alpha_{n}\right)=1$ for every $n$.

The lack of continuity of the scale might be remedied by considering the coarsest group topology, $\mathscr{T}$ say, on $\operatorname{Aut}(G)$ finer than the Braconnier topology and for which the scale is continuous. (It has been pointed out by Christian Rosendal that, when $G$ is second countable, $\mathscr{T}$ cannot be Polish if it is strictly finer than the Braconnier topology.) It would be desirable to have a more direct definition of $\mathscr{T}$. Even more desirable would be to have a directly defined topology on $\operatorname{End}(G)$ for which $\mathscr{T}$ is the subspace topology on $\operatorname{Aut}(G)$. In analogy with the ring of operators on a normed space and its open group of invertible operators, we might also ask whether $\operatorname{Aut}(G)$ open in this topology?

\section{Möller's "Spectral Radius" Formula}

The scale may be calculated without finding a minimising subgroup.

Theorem 2. [Spectral radius] Let $V$ be any compact open subgroup of $G$ and $\alpha$ be in End $(G)$. Then, the scale of $\alpha$ is equal to

$$
s(\alpha)=\lim _{n \rightarrow \infty}\left[\alpha^{n}(V): \alpha^{n}(V) \cap V\right]^{\frac{1}{n}}
$$

This formula is referred to as the "spectral radius formula" because of the similarity with the formula of the same name for linear operators on normed spaces. It turns out that the scale of an element $x$ in a t.d.l.c. group $G$ is exactly the spectral radius of the operator of translation by $x$ on a certain normed convolution algebra on $G$, see [14]. The formula was proved for automorphisms by R. G. Möller in ([3] Theorem 7.7) and extended to endomorphisms in [9, Proposition].

The spectral radius formula is illustrated by the next two examples. 
Example 2. Let $\mathcal{T}_{q+1}$ be the regular tree in which every vertex has valency $q+1$ and let $G=$ Aut $\left(\mathcal{T}_{q+1}\right)$. It may be seen that $G$ is a topological group under the topology of pointwise convergence on vertices and that for each vertex, $v$, in $\mathcal{T}_{q+1}$ the stabiliser stab ${ }_{G}(v)$ is open. Since, moreover, stab ${ }_{G}(v)$ isomorphic as a topological group to the iterated wreath product $S_{q+1} \backslash S_{q}>S_{q} 2 \ldots$ and is therefore profinite, it follows that $G$ is a locally compact group. It is a totally disconnected group because, for each pair $x \neq y \in G$ we may choose $v \in V\left(\mathcal{T}_{q+1}\right)$ with $x . v \neq y . v$ and then the sets $\mathscr{U}_{1}=\{g \in G \mid g . v=x . v\}$ and $\mathscr{U}_{2}=\{g \in G \mid g . v \neq x . v\}$ are an open partition of $G$ with $x \in \mathscr{U}_{1}$ and $y \in \mathscr{U}_{2}$.

Every automorphism of $G$ is inner. This may be shown, see [15], by observing that $\left\{\operatorname{stab}_{G}(v) \mid v \in V\left(\mathcal{T}_{q+1}\right)\right\}$ is a set of maximal compact open subgroups of $G$ on which each automorphism, $\alpha$ say, acts, and that the tree $\mathcal{T}_{q+1}$ may be reconstructed from this set of subgroups. The action of $\alpha$ on $\left\{\operatorname{stab}_{G}(v) \mid v \in V\left(\mathcal{T}_{q+1}\right)\right\}$ thus induces an automorphism, $x_{\alpha}$, of $\mathcal{T}_{q+1}$ and it may be seen that $\alpha$ is equal to the inner automorphism of conjugation by $x_{\alpha}$.

Consider $x \in G$ and the inner automorphism $\alpha_{x}: g \mapsto x g x^{-1}$. Let $U=G_{v}$ for some vertex $v$. As described in [15], there are two cases: $x$ could have finite orbits in $\mathcal{T}_{q+1}$, in which case it is called elliptic; or $x$ could have infinite orbits in $\mathcal{T}_{q+1}$, in which case it is called hyperbolic.

$x$ is elliptic. In this case, $\left\{\alpha_{x}^{n}(U) \mid n \geq 0\right\}=\left\{\operatorname{stab}_{G}\left(x^{n} . v\right) \mid n \geq 0\right\}$ is finite and hence the set of indices $\left.\overline{\left[\alpha_{x}^{n}(U): \alpha_{x}^{n}\right.}(U) \cap U\right]$ is bounded. Then,

$$
s(x)=\lim _{n \rightarrow \infty}\left[\alpha_{x}^{n}(U): \alpha_{x}^{n}(U) \cap U\right]^{\frac{1}{n}}=1 .
$$

$x$ is hyperbolic. In this case, $x$ is a translation along a geodesic path $\ell$, where a vertex $w$ is on $\ell$ if the distance $\overline{\text { from } w}$ to $x . w$ is a minimum. Let this minimum distance be $d$, so that $x$ translates $\ell$ by distance $d$. Let $w$ be the closest vertex on $\ell$ to the given vertex $v$. Then, $x . w$ is the closest vertex on $\ell$ to $x . v$ and the path from $v \rightarrow w \rightarrow x . w \rightarrow x . v$ is the shortest path from $v$ to $x . v$ and has length $d+2 c$, where $c$ is the distance from $v$ to $\ell$. Since the index $\left[\operatorname{stab}_{G}\left(u_{1}\right): \operatorname{stab}_{G}\left(u_{1}\right) \cap \operatorname{stab}_{G}\left(u_{2}\right)\right]$ is equal to $(q+1) q^{d\left(u_{1}, u_{2}\right)-1}$ for any two vertices $u_{1}$ and $u_{2}$, as may be seen by an application of the Orbit-Stabiliser Theorem, it follows that

$$
\left[\alpha_{x}^{n}(U): \alpha_{x}^{n}(U) \cap U\right]=\left[\operatorname{stab}_{G}\left(x^{n} . v\right), \operatorname{stab}_{G}\left(x^{n} . v\right) \cap \operatorname{stab}_{G}(v)\right]=(q+1) q^{d n+2 c-1} .
$$

Hence

$$
s(x)=\lim _{n \rightarrow \infty}\left((q+1) q^{d n+2 c-1}\right)^{\frac{1}{n}}=q^{d}
$$

Example 3. Let $G=F^{\mathbb{Z}}$, where $F$ is a non-trivial finite group, and equip $G$ with the product topology and the pointwise product. Then, $G$ is a compact group. Hence every endomorphism of $G$ has scale 1 because $G$ itself is a compact subgroup invariant under the endomorphism.

Let $\alpha$ the shift automorphism $\alpha(f)_{n}=f_{n+1}$. Consider the subgroup

$$
U_{K}=\left\{f \in F^{\mathbb{Z}} \mid f(k)=1_{F} \text { for } 0 \leq k<K\right\}
$$

An easy calculation shows that

$$
\left[\alpha^{n}\left(U_{K}\right): \alpha^{n}\left(U_{K}\right) \cap U_{K}\right]= \begin{cases}|F|^{n}, & \text { if } 0 \leq n \leq K \\ |F|^{K}, & \text { if } n>K\end{cases}
$$

Hence we find, as expected, that

$$
s(\alpha)=\lim _{n \rightarrow \infty}|F|^{\frac{K}{n}}=1
$$

\section{Identifying Minimising Subgroups}

Minimising subgroups have a structural characterisation which may be used to calculate the scale. Moreover, the proof of this characterisation involves a procedure for finding minimising subgroups. 
The characterisation and the procedure for finding minimising subgroups will now be described and then illustrated in several examples.

The characterisation of minimising subgroups for $\alpha \in \operatorname{End}(G)$ involves the following two subgroups defined for any compact and open subgroup $V$ of $G$.

$$
\begin{aligned}
& V_{+}=\left\{x \in V \mid \exists\left\{x_{n}\right\}_{n \in \mathbb{N}} \subset V \text { with } x_{0}=x \text { and } x_{n}=\alpha\left(x_{n+1}\right) \text { for each } n \in \mathbb{N}\right\} \\
& V_{-}=\left\{x \in V \mid \alpha^{n}(x) \in V \text { for all } n \in \mathbb{N}\right\} .
\end{aligned}
$$

The sequence $\left\{x_{n}\right\}_{n \in \mathbb{N}}$ appearing in the definition of $V_{+}$is a "history" of $x$ as $\alpha$ is iterated and the condition for $x$ to be in $V_{+}$is that this history is contained in $V$. This history need not be unique because $\alpha$ need not be injective, and it is not required that all histories of $x$ lie in $V$. The condition for $x$ to be in $V_{-}$is that the "future" of $x$ when $\alpha$ is iterated should lie in $V$.

In what follows, it is important to note that $\alpha\left(V_{+}\right) \geq V_{+}$. Hence, $\bigcup_{n \in \mathbb{N}} \alpha^{n}\left(V_{+}\right)$is an increasing union of subgroups of $G$ and is therefore itself a subgroup.

Theorem 3 (The structure of minimising subgroups). Let $\alpha \in \operatorname{End}(G)$ and $V$ be a compact open subgroup of $G$. Then, $V$ is minimising for $\alpha$ if and only if

TA: $V=V_{+} V_{-}$

TB1: $V_{++}:=\bigcup_{n \in \mathbb{N}} \alpha^{n}(V)$ is closed and

TB2: the sequence of integers $\left\{\left[\alpha^{n+1}\left(V_{+}\right): \alpha^{n}\left(V_{+}\right)\right] \mid n \in \mathbb{N}\right\}$ is constant.

In this case, $s(\alpha)=\left[\alpha\left(V_{+}\right): V_{+}\right]$.

Definition 2. A subgroup $V$ is tidy above for $\alpha$, if it satisfies condition TA; tidy below if it satisfies conditions TB1 and TB2; and is tidy if it is tidy above and below.

The condition TB2 is redundant if $\alpha$ is injective because it follows from the other conditions in that case. Hence, this condition did not appear in [4], which deals with automorphisms only.

\subsection{The Tidying Procedure}

This section describes a three-step procedure which takes as input a general compact open subgroup, $U$, and produces a subgroup tidy for $\alpha$. The procedure is given effect by the following three propositions.

Proposition 1 (Step 1). Let $U \leq G$ be compact and open and $\alpha \in \operatorname{End}(G)$. Then, there is $N \in \mathbb{N}$ such that the subgroup $U_{-N}=\bigcap_{n=0}^{N} \alpha^{-n}(U)$ is tidy above for $\alpha$. For this $N$, we have

$$
\left[\alpha\left(U_{-N}\right): \alpha\left(U_{-N}\right) \cap U_{-N}\right] \leq[\alpha(U): \alpha(U) \cap U] .
$$

Remark 1. The proof of the proposition involves forming the decreasing sequence of subgroups $\left\{U_{k}\right\}_{k \in \mathbb{N}}$, where

$$
U_{k}=\left\{u \in U \mid \exists u_{0}, u_{1}, \ldots, u_{k} \in U \text { with } u_{n}=\alpha\left(u_{n+1}\right) \text { for } 0 \leq n<k \text { and } u_{0}=u\right\} .
$$

Then, $\bigcap_{k \in \mathbb{N}} U_{k}=U_{+}$and $N$ is the first $k$ such that $U_{k} \subset U_{+}\left(U \cap \alpha^{-1}(U)\right)$. That such $k$ exists follows from compactness of $U_{k}$ and the fact that $U_{+}\left(U \cap \alpha^{-1}(U)\right)$ is an open neighbourhood of $U_{+}$. This step involves cutting down $U$ to the subgroup $U_{-N}$ and motivates the name 'tidy above' for the factorisation property satisfied by $U_{-N}$.

The next two steps involve ensuring that the group is 'tidy below' by including in it the compact subgroup identified in the next proposition. 
Proposition 2 (Step 2). Suppose that $V \leq G$ is tidy above for $\alpha$ and define

$$
\mathcal{L}_{V}=\left\{x \in G \mid \exists m, n \in \mathbb{N} \text { with } x \in \alpha^{m}\left(V_{+}\right) \text {and } \alpha^{n}(x) \in V_{-}\right\} .
$$

Then, $\overline{\mathcal{L}_{V}}$ is compact and $\alpha$-stable.

Local compactness of $G$ is again important in the proof of this proposition.

The third proposition combines $V$ with $\mathcal{L}_{V}$ to form a subgroup that is tidy for $\alpha$. It is not enough to simply multiply the two subgroups because that might not be a subgroup. The subgroup generated by $V$ and $\mathcal{L}_{V}$ might not be compact, and so that method of combining the subgroups will not work either.

Proposition 3 (Step 3). Suppose that $V \leq G$ is tidy above for $\alpha$ and define

$$
\widetilde{V}=\left\{x \in V \mid x \mathcal{L}_{V} \subset \mathcal{L}_{V} V\right\} \text { and } W=\widetilde{V} \mathcal{L}_{V}
$$

Then $W$ is "a compact open subgroup of $G$ that is tidy for $\alpha$ " and

$$
[\alpha(W): \alpha(W) \cap W] \leq[\alpha(V): \alpha(V) \cap V]
$$

\subsection{Tidy Subgroups Are Minimising and Conversely}

These three steps take a general compact open subgroup and modify it to produce a tidy subgroup. The next result implies that this subgroup is minimising for $\alpha$.

Theorem 4. The index $[\alpha(W): \alpha(W) \cap W]$ is the same for all compact open subgroups tidy for $\alpha$.

To prove the claim that tidy subgroups are minimising, suppose that the compact open subgroup $U$ is minimising and apply the tidying procedure to $U$. Then, the subgroup $W$ so produced is tidy and $[\alpha(W): \alpha(W) \cap W] \leq[\alpha(U): \alpha(U) \cap U]$. Since $U$ was already minimising, we conclude that $W$ is minimising and hence so are all subgroups tidy for $\alpha$.

That all minimising subgroups are tidy may be seen by noting that the inequalities in Propositions 1 and 3 are equalities if and only if the group already satisfies the relevant tidiness condition. Hence, if $U$ is already minimising, the tidying procedure does not alter it and $U$ is therefore tidy.

\subsection{Tidy Subgroups and the Scale in Examples}

Theorem 3 and the notion of tidy subgroup will now be illustrated by using them to compute the scale for the same automorphisms as in the previous section, as well as for some additional examples.

Example 2 (Revisited), let $G=\operatorname{Aut}\left(\mathcal{T}_{q+1}\right)$ and $\alpha=\alpha_{x}$ be an automorphism as before. The tidying procedure will be applied with $U=\operatorname{stab}_{G}(v)$ for an arbitrary vertex $v$ in $\mathcal{T}_{q+1}$. For this, note that $\alpha_{x}^{n}(U)=\operatorname{stab}_{G}\left(x^{n} \cdot v\right)$.

Since $\alpha_{x}$ is an automorphism, and is in particular injective, the subgroup $U_{k}$ defined as in Equation (2), is equal to $\bigcap_{j=0}^{k} \alpha^{j}(U)$ and the subgroup $U_{+}$defined as in Equation (1) is equal to $\bigcap_{j=0}^{\infty} \alpha^{j}(U)$. Hence, $U_{k}$ is the fixator of the vertices $v, x . v, \ldots, x^{k} . v$ and $U_{+}$is the fixator of the vertices $x^{n} . v, n \in \mathbb{N}$. As before, the cases when $x$ is elliptic and when it is hyperbolic are treated separately.

$x$ is elliptic. The orbit $x^{n} . v, n \in \mathbb{Z}$ is finite and so there is $k$ such that $\left\{v, x . v, \ldots, x^{k} . v\right\}$ is equal to this orbit. Choosing $N=k$, we then have that $U_{+}=U_{k}$ and it is easily seen that $U_{-}$is equal to this subgroup as well. Hence, putting

$$
V=U_{k}=\operatorname{stab}_{G}(v) \cap \operatorname{stab}_{G}(x . v) \cap \cdots \cap \operatorname{stab}_{G}\left(x^{k} . v\right),
$$


we have that $\alpha_{x}(V)=V=V_{+}=V_{-}$. Hence, $V$ is tidy above. It follows that $V_{++}=V$ as well and is closed. Therefore, $V$ is tidy for $\alpha_{x}$. Of course,

$$
\left[\alpha_{x}(V): \alpha_{x}(V) \cap V\right]=[V: V]=1
$$

$x$ is hyperbolic. As before, let $\ell$ be the axis for $x$, suppose that $x$ translates along $\ell$ through distance $d$, let $c$ be the distance from $v$ to $\ell$, and let $w$ be the vertex on $\ell$ that is closest to $v$. In addition, denote the neighbour of $w$ closest to $x . v$ by $w^{+}$and the neighbour of $w$ closest to $x^{-1} . v$ by $w^{-}$.

The subgroup $U$ itself is not tidy for $\alpha_{x}$. To see this, note that since $U_{+}$fixes all vertices $x^{n} . v$ with $n \geq 0$, it fixes $w$ and $w^{+}$as well. Similarly, $U_{-}$fixes $w$ and $w^{-}$. Hence $U \neq U_{+} U_{-}$if $v \neq w$ and $U$ is not tidy above. The same conclusion holds even when $v=w$ because, while $U$ acts as the full permutation group $S_{q+1}$ on the $q+1$ neighbours of $w, U_{+}$and $U_{-}$each fix one of the neighbours and $S_{q+1}$ is not equal to the product of two such subgroups. We see too that, since $x$ fixes every vertex on the path from $v$ to $x . v$, the same calculation as in the earlier discussion yields that

$$
\left[\alpha_{x}(U): \alpha_{x}(U) \cap U\right]=(q+1) q^{d+2 c-1},
$$

which is strictly greater than $q^{d}$. Hence, $U$ is not minimising.

Step 1 The subgroup $U_{-1}=U \cap \alpha_{x}^{-1}(U)$ is tidy above however. Setting $V=U_{-1}$, we have that $V$ fixes all vertices on the path from $w$ to $x^{-1} . w$. Hence, the Tits independence property for $\operatorname{Aut}\left(\mathcal{T}_{q+1}\right)$ implies that $V=H_{+} H_{-}$, where $H_{+}$and $H_{-}$are the fixators in $V$ of the components $\mathcal{S}_{+}$and $\mathcal{S}_{-}$ of $\mathcal{T}_{q+1}$ formed when the path from $w$ to $x^{-1} . w$ is deleted and containing $x^{-1} . w$ and $w$ respectively. Since $H_{ \pm} \leq V_{ \pm}$, it follows that $V$ is tidy above. The index we are interested in may be calculated to be

$$
\left[\alpha_{x}(V): \alpha_{x}(V) \cap V\right]=\left\{\begin{array}{ll}
(q-1) q^{d+c}, & \text { if } v \notin \ell \\
q^{d}, & \text { if } v \in \ell^{\prime}
\end{array},\right.
$$

which is strictly less than that found in (3). However, it is not the minimum value calculated using the spectral radius formula unless $v$ happens to lie on $\ell$, or $q=2$ and $c=1$. The second and third steps of the tidying procedure must therefore be implemented to find a minimising subgroup.

Carrying out these steps will require labelling some more vertices of $\mathcal{T}_{q+1}$. Denote the set of $q+1$ neighbours of $w$ by $N(w)$ and similarly for $x^{-1} . w$. Recall that $w^{+}$and $w^{-}$are neighbours of $w$ on $\ell$, and denote by $w^{o}$ the neighbour of $w$ lying on the path from $v$ to $w$. Then $x^{-1} . w^{+}, x^{-1} . w^{-}$and $x^{-1} . w^{o}$ are neighbours of $x^{-1} . w: x^{-1} . w^{+}$is the neighbour closest to $w ; x^{-1} . w^{-}$is closest to $x^{-2} . w$; and $x^{-1} . w^{o}$ lies on the path from $x^{-1} . v$ to $x^{-1} . w$. The subgroup $V$ found in the previous paragraph fixes all vertices on $\ell$ between $w$ and $x^{-1} . w$ as well as the vertices on the paths joining $v$ to $w$ and $x^{-1} . v$ to $x^{-1} . w$, that is, all vertices on the path from $v$ to $x^{-1} . v$. In particular, $V$ acts on $N(w)$ by fixing $w^{o}$ and $w^{-}$and as the full symmetric group on $N(w) \backslash\left\{w^{o}, w^{-}\right\}$; and acts on $N\left(x^{-1} . w\right)$ by fixing $x^{-1} . w^{o}$ and $x^{-1} . w^{+}$and as the full symmetric group on $N\left(x^{-1} . w\right) \backslash\left\{x^{-1} . w^{o}, x^{-1} . w^{+}\right\}$.

Step 2 The definition of $\mathcal{L}_{V}$ in Proposition 2 implies that $y$ belongs to this subgroup if $\alpha_{x}^{n}(y) \in V$ for all but finitely many $n$. Hence, tree automorphisms in $\mathcal{L}_{V}$ fix all the vertices of $\ell$ and all vertices on paths joining $x^{n} . v$ to $\ell$ except for finitely many $n$. The action of $\mathcal{L}_{V}$ on $N(w)$ thus fixes $w^{+}$and $w^{-}$and permutes vertices in $N(w) \backslash\left\{w^{-}, w^{+}\right\}$arbitrarily; and its action on $N(x . w)$ fixes $x^{-1} . w^{-}$and $x^{-1} . w^{+}$ and permutes vertices in $N(x . w) \backslash\left\{x^{-1} . w^{+}, x^{-1} . w^{-}\right\}$arbitrarily. That the closure of $\mathcal{L}_{V}$ is compact as claimed in Proposition 2 may be seen by observing that this closure is the fixator of all vertices on $\ell$.

Step 3 That the product $\mathcal{L}_{V} V$ is not a group may be seen by considering its action on $N(w)$. While $V$ and $\mathcal{L}_{V}$ both fix $w$, we have that $\mathcal{L}_{V}$ fixes $w^{+}$and $w^{-}$and acts as $\left.\operatorname{Sym}(N(w)) \backslash\left\{w^{-}, w^{+}\right\}\right)$on the 
remaining vertices in $N(w)$; and $V$ fixes $w^{0}$ and $w^{-}$and acts as $\left.\operatorname{Sym}(N(w)) \backslash\left\{w^{0}, w^{-}\right\}\right)$on the remaining vertices; but the product

$$
\left.\operatorname{Sym}(N(w)) \backslash\left\{w^{-}, w^{+}\right\}\right) \operatorname{Sym}\left(N(w) \backslash\left\{w^{o}, w^{-}\right\}\right)
$$

is not a subgroup of $\operatorname{Sym}(N(w))$. In the present example, $\left\langle\mathcal{L}_{V}, V\right\rangle$ is compact and equal to the fixator of the path from $w$ to $x^{-1} . w$, which is tidy for $\alpha_{x}$. We shall see, however, that the procedure described in Proposition 3 produces a different tidy subgroup. According to this procedure, define $\widetilde{V}=\left\{g \in V \mid g \mathcal{L}_{V} \subset \mathcal{L}_{V} V\right\}$. To determine $\widetilde{V}$, we apply the following lemma about finite permutation groups to the subgroups $\left.\operatorname{Sym}(N(w)) \backslash\left\{w^{0}, w^{-}\right\}\right)$and $\left.\operatorname{Sym}(N(w)) \backslash\left\{w^{+}, w^{-}\right\}\right)$ of $\left.\operatorname{Sym}(N(w)) \backslash\left\{w^{-}\right\}\right)$.

Lemma 1. Let $S_{q}$ denote the permutation group $S_{q}=\operatorname{Sym}(\{1,2, \ldots, q\})$. Then,

$$
\left\{\pi \in \operatorname{stab}_{S_{q}}(1) \mid \pi \operatorname{stab}_{S_{q}}(q) \subset \operatorname{stab}_{S_{q}}(q) \operatorname{stab} b_{S_{q}}(1)\right\}=\operatorname{stab}_{S_{q}}(1, q) .
$$

Proof. Note that no permutation in $\operatorname{stab}_{S_{q}}(q) \operatorname{stab}_{S_{q}}(1)$ sends 1 to $q$. On the other hand, if $\pi \in \operatorname{stab}_{S_{q}}(1)$ and does not fix $q$, then there is $j \in\{2, \ldots, q-1\}$ such that $\pi(j)=q$ and there is $\sigma \in \operatorname{stab}_{S_{q}}(q)$ such that $\sigma(1)=j$. Then $\pi \sigma(1)=q$ and is not in $\operatorname{stab}_{S_{q}}(q) \operatorname{stab}_{S_{q}}(1)$.

It follows from Lemma 1 that

$$
\widetilde{V} \subset\left\{g \in V \mid g \text { fixes } w^{o}, w^{-} \text {and } w^{+}\right\} \cap\left\{g \in V \mid g \text { fixes } x^{-1} . w^{o}, x^{-1} . w^{-} \text {and } x^{-1} . w^{+}\right\} .
$$

Since elements of $V$ fix $w^{o}, w^{-}, x^{-1} \cdot w^{+}$and $x^{-1} \cdot w^{o}$ already,

$$
\widetilde{V} \subset\left\{g \in V \mid g \text { fixes } w^{+} \text {and } x^{-1} \cdot w^{-}\right\} .
$$

It is easily seen that all elements of $V$ fixing $w^{+}$and $x^{-1} . w^{-}$belong to $\widetilde{V}$. Hence $\widetilde{V}=\operatorname{stab}_{V}\left(w^{+}\right) \cap$ $\operatorname{stab}_{V}\left(x^{-1} \cdot w^{-}\right)$. Then,

$$
W=\mathcal{L}_{V} \widetilde{V}=\operatorname{stab}_{G}\left(w^{+}\right) \cap \operatorname{stab}_{G}\left(x^{-1} \cdot w^{-}\right),
$$

that is, elements of $W$ fix all vertices on the axis $\ell$ between $x^{-1} . w^{-}$and $w^{+}$.

To compute the scale of $\alpha_{x}$ using this tidy subgroup $W$, observe that $\alpha_{x}(W)$ fixes all vertices on $\ell$ between $w^{-}$and $x . w^{+}$and $\alpha_{x}(W) \cap W$ fixes all vertices between $x^{-1} \cdot w^{-}$and $x . w^{+}$. The distance on $\ell$ between $x^{-1} . w^{-}$and $w^{-}$is $d$, and the orbit of $x^{-1} . w^{-}$under $\alpha_{x}(W)$ therefore has order $q^{d}$. Hence,

$$
s\left(\alpha_{x}\right)=\left[\alpha_{x}(W): \alpha_{x}(W) \cap W\right]=q^{d} .
$$

Regular trees are a particular type of building — see [16] for the definition—and automorphism groups of locally finite buildings are totally disconnected, locally compact groups. The calculation of the scale in terms of geometric data describing the building could also be carried out by a similar approach to that used for trees.

Example 3 (Revisited), let $G=F^{\mathbb{Z}}$ for some finite group $F$ and $\alpha$ be the shift automorphism as before. It has already been remarked that $G$ is compact and invariant under $\alpha$ and so $s(\alpha)=1$. When the tidying procedure is applied with $U=G$ there is no change: in Step 1, we have $N=0$ and so $V=U$ in the next step; in Step 2, $\mathcal{L}_{V}=V$; and in Step 3, $\widetilde{V}=V$ and $W=V=U$.

The tidying procedure will be illustrated by applying it to the compact open subgroup

$$
U_{X}=\left\{f \in F^{\mathbb{Z}} \mid f(k)=1_{F} \text { for all } k \in X\right\},
$$


where $X$ is a finite subset of $\mathbb{Z}$. When $X=\{0,1, \ldots, K-1\}$, we recover the subgroup $U_{K}$ considered previously.

Step 1 Since $\alpha$ is an automorphism, the group $\left(U_{X}\right)_{n}$ defined in Proposition 1 is equal to $\bigcap_{k=0}^{n} \alpha^{k}\left(U_{X}\right)$ and the number $N$ whose existence is guaranteed by the proposition depends on $X$.

In the case when $X=\{0,1, \ldots, K-1\}$ the subgroup $U_{X}=U_{K}$ is already tidy above and $N=0$. To see this, note that

$$
\left(U_{K}\right)_{+}=\left\{f \in F^{\mathbb{Z}} \mid f(k)=1_{F} \text { if } k<K\right\} \text { and }\left(U_{K}\right)_{-}=\left\{f \in F^{\mathbb{Z}} \mid f(k)=1_{F} \text { if } k \geq 0\right\} .
$$

In this case, we have $\left[\alpha\left(U_{K}\right): \alpha\left(U_{K}\right) \cap U_{K}\right]=|F|$.

For another case, suppose that $X=\{0,1,5,6,7,8\}$. Then,

$$
\alpha^{n}\left(U_{X}\right)=\left\{f \in F^{\mathbb{Z}} \mid f(k)=1_{F} \text { if } k \in X-n\right\} .
$$

Hence, $\alpha\left(U_{X}\right) \cap U_{X}=\left\{f \in F^{\mathbb{Z}} \mid f(k)=1_{F}\right.$ if $\left.k \in X \cup(X-1)\right\}$. In other words,

$$
\begin{aligned}
\alpha\left(U_{X}\right) & =\left\{f \in F^{\mathbb{Z}} \mid f(k)=1_{F} \text { if } k \in\{-1,0,4,5,6,7\}\right\} \text { and } \\
\alpha\left(U_{X}\right) \cap U_{X} & =\left\{f \in F^{\mathbb{Z}} \mid f(k)=1_{F} \text { if } k \in\{-1,0,1,4,5,6,7,8\}\right\}
\end{aligned}
$$

and $\left[\alpha\left(U_{X}\right): \alpha\left(U_{X}\right) \cap U_{X}\right]=|F|^{2}$. Moreover,

$$
\left(U_{X}\right)_{+}=\left\{f \in F^{\mathbb{Z}} \mid f(k)=1_{F} \text { if } k<9\right\} \text { and }\left(U_{K}\right)_{-}=\left\{f \in F^{\mathbb{Z}} \mid f(k)=1_{F} \text { if } k \geq 0\right\}
$$

and $\left(U_{X}\right)_{+}\left(U_{X}\right)_{-} \neq U_{X}$. Similar calculations apply for $\left(U_{X}\right)_{1}=\alpha\left(U_{X}\right) \cap U_{X}$ and $\left(U_{X}\right)_{2}=\alpha^{2}\left(U_{X}\right) \cap$ $\alpha\left(U_{X}\right) \cap U_{X}$. However,

$$
\left(U_{X}\right)_{3}=\left\{f \in F^{\mathbb{Z}} \mid f(k)=1_{F} \text { if }-3 \leq k \leq 8\right\},
$$

which is tidy above, and $\left[\alpha\left(\left(U_{X}\right)_{3}\right): \alpha\left(\left(U_{X}\right)_{3}\right) \cap\left(U_{X}\right)_{3}\right]=|F|$.

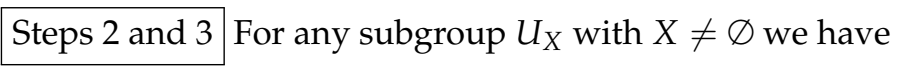

$$
\mathcal{L}_{U_{X}}=\left\{f \in F^{\mathbb{Z}} \mid f(k)=1_{F} \text { for all but finitely many } k\right\}
$$

and $\mathcal{L}_{U_{X}} U_{X}=G$.

Lie groups over local fields are totally disconnected and locally compact as well, and the scale of elements in such groups, that is, of inner automorphisms of the groups, was computed by H. Glöckner in [17]. His were the first calculations of the scale for groups that went beyond the basic cases seen in the previous examples.

Example 4. Let $G$ be a Lie group over the field $\mathbb{Q}_{p}$ of p-adic numbers. Glöckner does not use the tidying procedure to find subgroups tidy for $x$ (that is, for $\alpha_{x}$ ) but instead describes $V_{+}$and $V_{-}$directly in terms of the normal form of the Lie algebra automorphism $\operatorname{Ad}_{x}$ and calculates $s(x)$ in terms of eigenvalues of $\operatorname{Ad}_{x}$ (in a finite extension of $\mathbb{Q}_{p}$ ). This correspondence between the scale and tidy subgroups on one hand and eigenvalues and eigenspaces on the other is evidence that scale techniques are a substitute for Lie algebra techniques when studying t.d.l.c. groups that are not Lie groups over local fields.

The main ideas in [17] may be sketched as follows. Assume that $V$ is tidy for $x$, then $V_{++}$is closed and so is a Lie subgroup of $G$. Moreover, $V_{+}$is an open subgroup of $V_{++}$and $s(x)=\Delta\left(I_{x}\right)$, where $I_{x}$ is the automorphism of $V_{++}$induced by conjugation by $x$ and $\Delta$ is the module function on automorphisms. The module of this automorphism of $V_{++}$is then equal to the module of the automorphism $L\left(I_{x}\right)$ induced on the Lie 
algebra of $V_{++}$. Glöckner then describes this Lie algebra as a subalgebra of $L(G)$ (the Lie algebra of $G$ ) in what he calls the contraction decomposition of $\mathrm{Ad}_{x}$. This decomposition applies to any linear automorphism $\phi$ of $a$ finite-dimensional vector space, $L$, over a local field of characteristic 0 , and expresses $L$ as

$$
L=L_{p} \oplus L_{0} \oplus L_{m}
$$

where

$$
\begin{aligned}
L_{p} & :=\left\{y \in L: \phi^{-k}(y) \rightarrow 0 \text { as } k \rightarrow \infty\right\} \\
L_{m} & :=\left\{y \in L: \phi^{k}(y) \rightarrow 0 \text { as } k \rightarrow \infty\right\} \text { and } \\
L_{0} & :=\left\{y \in L:\left\{\left\|\phi^{k}(y)\right\|\right\}_{k \in \mathbb{Z}} \text { is bounded }\right\} .
\end{aligned}
$$

Glöckner bases this decomposition on a variation on ([18] Lemma 3.4). Applying it when L is the Lie algebra of $G$ and $\phi=\operatorname{Ad}_{x}$, he finds that $L_{p} \oplus L_{0}$ is a subalgebra isomorphic to the Lie algebra of $V_{++}$. Using this decomposition, Glöckner shows in ([17] Corollary 3.6) that, if $G$ is a Lie group over a local field, $K$, of characteristic 0 and $x$ is in $G$, then

$$
s(x)=\prod_{\left|\lambda_{i}\right| \geq 1}\left|\lambda_{i}\right|
$$

where $\lambda_{i}$ are the roots of the characteristic polynomial of $\operatorname{Ad}_{x}$ in a splitting field, $K^{\prime}$, for this polynomial and $|\cdot|$ is the unique extension to $K^{\prime}$ of absolute value on $K$.

Glöckner thus reduces the computation of the scale to finding eigenvalues and avoids the need to find tidy subgroups. He gives more explicit formulx for the scale on linear algebraic groups. The formulx and their relation to the methods for computing the scale previously discussed may be illustrated with the case when the group is $\mathrm{GL}\left(2, \mathbb{Q}_{p}\right)$ and the element $x$ has the property that its characteristic polynomial splits over $\mathbb{Q}_{p}$, in which case there is a basis for $\mathbb{Q}_{p}^{2}$ with respect to which $x$ has a diagonal matrix with entries $\lambda_{1}, \lambda_{2}$, the eigenvalues of $x$. Consider the compact, open subgroup $U=\mathrm{GL}\left(2, \mathbb{Z}_{p}\right)$. Note that the condition that $U$ is a group forces the determinant of each element of $U$ to have p-adic absolute value equal to 1.

Applying powers of $\alpha_{x}$ to $U$ yields that

$$
\begin{aligned}
\alpha_{x}^{n}(U) & =\left\{\left(\begin{array}{cc}
a & \left(\lambda_{1} \lambda_{2}^{-1}\right)^{n} b \\
\left(\lambda_{1}^{-1} \lambda_{2}\right)^{n} c & d
\end{array}\right): a, b, c, d \in \mathbb{Z}_{p},|a d-b c|_{p}=1\right\} \\
& =\left\{\left(\begin{array}{cc}
a & p^{-k n} b \\
p^{k n} c & d
\end{array}\right): a, b, c, d \in \mathbb{Z}_{p},|a d-b c|_{p}=1\right\},
\end{aligned}
$$

where $\left|\lambda_{1} \lambda_{2}^{-1}\right|=p^{k}$. Thus, if $k=0$, then $\alpha_{x}(U)=U$ and $s\left(\alpha_{x}\right)=s(x)=1$. Suppose that $k>0$. (The case when $k<0$ is similar.) Then,

$$
\alpha_{x}^{n}(U) \cap U=\left\{\left(\begin{array}{cc}
a & b \\
p^{k n} c & d
\end{array}\right): a, b, c, d \in \mathbb{Z}_{p},|a d-b c|_{p}=|a d|_{p}=1\right\}
$$

and it may be calculated that

$$
\left[\alpha_{x}^{n}(U): \alpha_{x}^{n}(U) \cap U\right]=(p+1) p^{k n-1} .
$$

Hence, by Theorem 2, $s(x)=\lim _{n \rightarrow \infty}\left((p+1) p^{k n-1}\right)^{\frac{1}{n}}=p^{k}=\left|\lambda_{1} \lambda_{2}^{-1}\right|$, which is the same value as given in the last example in ([17] Corollary 3.6).

The subgroup $U$ is not tidy above for $\alpha_{x}$ when $k>0$ because, as follows from (5),

$$
U_{+}=\left\{\left(\begin{array}{ll}
a & b \\
0 & d
\end{array}\right): a, b, d \in \mathbb{Z}_{p},|a d|_{p}=1\right\} \text { and } U_{-}=\left\{\left(\begin{array}{ll}
a & 0 \\
c & d
\end{array}\right): a, c, d \in \mathbb{Z}_{p},|a d|_{p}=1\right\}
$$


and the element $u=\left(\begin{array}{ll}0 & 1 \\ 1 & 0\end{array}\right)$ belongs to $U$ but not to $U_{+} U_{-}$.

Step 1 of the tidying procedure. The subgroup

$$
V=U \cap \alpha_{x}(U)=\left\{\left(\begin{array}{cc}
a & b \\
p^{k} c & d
\end{array}\right): a, b, c, d \in \mathbb{Z}_{p},|a d|_{p}=1\right\}
$$

may be verified to be tidy above by showing that every element of $V$ is the product of an upper triangular and a lower triangular matrix. Proposition 1 thus holds with $N=1$ in this case.

Steps 2 and 3 It may also be verified that

$$
V_{++}=\left\{\left(\begin{array}{ll}
a & b \\
0 & d
\end{array}\right): b \in \mathbb{Q}_{p}, a, d \in \mathbb{Z}_{p},|a d|_{p}=1\right\},
$$

which is a closed subgroup of $\mathrm{GL}\left(2, \mathbb{Q}_{p}\right)$, and hence that $V$ is also tidy below. That the tidying procedure terminates after the first step in this case is no accident: it is shown in ([19] Theorem 3.2) that that occurs for any automorphism $\alpha$ for which the contraction subgroup,

$$
\operatorname{con}(\alpha)=\left\{y \in G: \alpha^{n}(y) \rightarrow 1_{G} \text { as } n \rightarrow \infty\right\},
$$

is closed and it is shown in ([18] Theorem 3.5) that that is always so for automorphisms of p-adic Lie groups.

Glöckner has also calculated the scale in some cases for linear groups over a skew field, $K$, with positive characteristic, see [20]. He shows that, if $x$ is a diagonalisable element in $\operatorname{GL}(n, K), \operatorname{SL}(n, K), \operatorname{PGL}(n, K)$ or $\operatorname{PSL}(n, K)$, then the scale is given by the same formula as in the characteristic 0 case. He does so by writing down tidy subgroups for $\alpha_{x}$. In particular, he shows that, if the diagonal entries in $x$ are in order of decreasing modulus, then certain compact, open subgroups may be written as the product of their subgroup of upper triangular matrices with their subgroup of lower triangular matrices and that this implies tidiness above. Tidiness below is again satisfied automatically, as in the previous paragraph, because contraction subgroups for inner automorphisms are closed.

More is known about $p$-adic Lie groups than for general t.d.l.c. groups but a key question remains unanswered even for these groups. If an element $x$ in a t.d.l.c. group satisfies that $s(x)=1=s\left(x^{-1}\right)$, then subgroups tidy for $x$ are normalised by $x$ and, conversely, if $U$ is normalised by $x$, then $s(x)=$ $1=s\left(x^{-1}\right)$. A t.d.l.c. group $G$ is uniscalar if $s(x)=1$ for every $x \in G$ and it is shown in [21], relying on a result in [22], that a $p$-adic Lie group that is compactly generated and uniscalar has a compact, open normal subgroup. There are uniscalar t.d.l.c. groups having no compact, open normal subgroups which are compactly generated, see [23], and which are topologically simple, see [24]. However, no examples are known of uniscalar t.d.l.c. groups which are topologically simple and compactly generated, or which are topologically simple (of necessity not compactly generated) and $p$-adic Lie.

Another significant class of t.d.l.c. groups are the groups of almost automorphisms of trees introduced by Yu. Neretin, [25,26], and shown to be simple by C. Kapoudjian, [27]. Neretin groups are also studied in [28], where it is shown that they do not contain a lattice, and the notation used here conforms with that paper. The papers [29], on abstract commensurators, and [30], on 'germs of automorphisms' are also relevant. Neretin's groups are also the inspiration for the simple groups acting on trees recently constructed in [31].

Example 5. An almost automorphism of an infinite, locally finite tree $\mathcal{T}$ is a bijection on the vertices of $\mathcal{T}$ which preserves all but finitely many edge relations. The set of almost automorphisms forms a group under composition of bijections. This group has two subgroups which are important for this discussion: the 
group $A u t(\mathcal{T})$ of automorphisms of $\mathcal{T}$; and the group $F \operatorname{Sym}(V)$ of finite permutations of the vertices of $\mathcal{T}$. Fix a vertex $v$ in $\mathcal{T}$ and let $U=\operatorname{stab}_{A u t(\mathcal{T})}(v)$. Then, $U$ is a compact group under the subspace topology of $A u t(\mathcal{T})$ and each almost automorphism of $\mathcal{T}$ commensurates some open subgroup of $U$ to another open subgroup. Hence,

$\{x V \mid x$ an almost automorphism and $V$ an open subgroup of $U\}$

is a sub-base of a group topology on the group of almost automorphisms of $\mathcal{T}$. Since $U$ is open in this topology, the group of almost automorphisms is then a locally compact group. Since non-trivial elements of FSym $(V)$ cannot be in $U$, the subgroup FSym $(V)$ is closed in this topology and is easily seen to be normal as well. (It may be seen that $F \operatorname{Sym}(V)$ is the quasi-centre of the group of almost automorphisms, see [32] for the definition.) The quotient of the group of almost automorphisms by $\operatorname{Fym}(V)$ is therefore a locally compact group which will be denoted by $A A u t(\mathcal{T})$. It is this quotient group which will from now on be referred to as the group of almost automorphisms of $\mathcal{T}$.

Alternative but equivalent definitions of $A A u t(\mathcal{T})$ are used elsewhere. For example, in [28], two almost automorphisms of $\mathcal{T}$ are defined to be equivalent if they agree on the complement of some finite subtree of $\mathcal{T}$ and $A A u t(\mathcal{T})$ is defined to be the set of equivalence classes of almost automorphisms. Since each finite set of vertices in $\mathcal{T}$ spans a finite subtree, this is the same as the equivalence relation of two almost automorphisms agreeing modulo FSym $(V)$. Almost automorphisms of $\mathcal{T}$ may be seen to be equivalent if and only if the actions they induce on the boundary, $\partial \mathcal{T}$, of the tree agree. The group $A A u t(\mathcal{T})$ may thus be defined in terms of its action on $\partial \mathcal{T}$. In these terms, $A A u t(\mathcal{T})$ is the full group of the action on $A u t(\mathcal{T})$ on $\partial \mathcal{T}$.

Almost automorphisms of the rooted tree $\mathcal{T}_{q, r}$, in which the root has $r$ children and every other vertex has q children, have been studied extensively. In this notation, Neretin's group of almost automorphisms is $A A u t\left(\mathcal{T}_{2,2}\right)$. The group $A A u t\left(\mathcal{T}_{q, r}\right)$ has the Higman-Thompson group $G_{q, r}$, see [33,34], as a dense subgroup and elements of $G_{q, r}$ may be represented (non-uniquely) as pairs, $\left(\mathcal{F}_{1}, \mathcal{F}_{2}\right)$, of finite rooted subtrees of $\mathcal{T}_{q, r}$, see [33] or ([35] Section 3) for example. Since the scale is continuous, it therefore suffices, in order to compute the scale on $A A u t\left(\mathcal{T}_{q, r}\right)$, to compute it for elements represented by such pairs of finite trees. This calculation is intricate and the general case is not described in full detail here. Instead, the ideas will be illustrated by the calculation of $s(x)$ for one element $x$ in $G_{2,2}<A A u t\left(\mathcal{T}_{2,2}\right)$. Note, however, that this example only displays some of the intricacies arising in the calculation of the scale on $A A u t\left(\mathcal{T}_{q, r}\right)$.

Let $x$ be the element of $G_{2,2}$ (also known as Thompson's group $V$ ) described by the pair of trees $\left(\mathcal{F}_{1}, \mathcal{F}_{2}\right)$ in Figure 1. When $G_{2,2}$ is embedded in $A A u t\left(\mathcal{T}_{2,2}\right)$, this element denotes the almost automorphism which sends the vertices labelled $1, \ldots, 6$ in the tree on the left to the vertices with the corresponding labels $1^{\prime}, \ldots, 6^{\prime}$ in the tree on the right and copies across the subtrees below each of these vertices. Thus, the subtree of $\mathcal{F}_{1}$ whose root is the vertex on level 3 and labelled 1 is raised to a subtree with root on level 2, the subtrees with roots on level 3 and labelled 2 and 3 are copied across to two other subtrees with roots on level 3 , the subtrees with roots on level 4 and labelled 4 and 5 are raised to subtrees with roots on level 2 and 3 respectively, and the subtree with root on level 1 and labelled 6 is lowered to a vertex on level 3. 


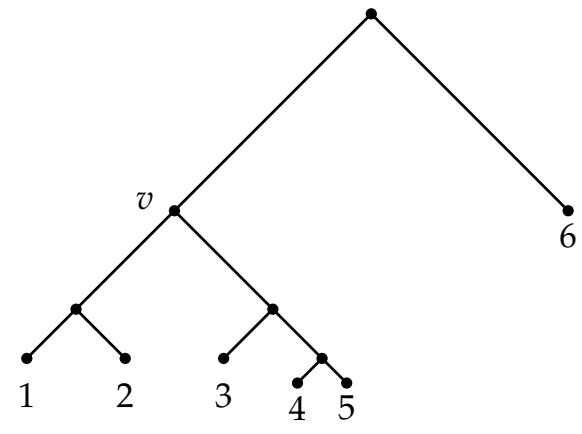

$\mathcal{F}_{1}$

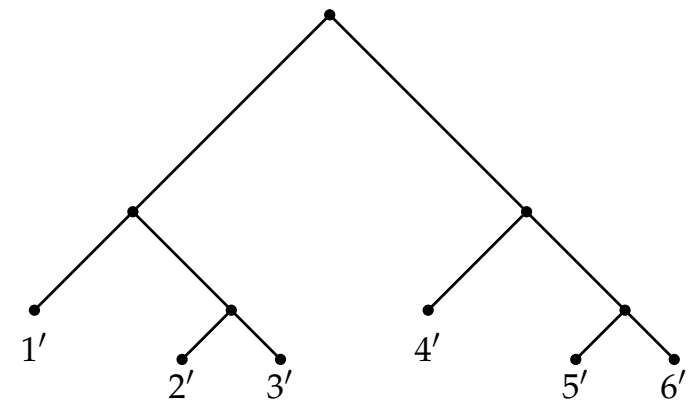

$\mathcal{F}_{2}$

Figure 1. Pair of trees for the element $x$ of Thompson's group $V$.

Let $U=\operatorname{Aut}\left(\mathcal{T}_{2,2}\right)$, so that $U$ is a compact, open subgroup of $A$ Aut $\left(\mathcal{T}_{2,2}\right)$, and let $\widetilde{U}$ be the subgroup of $U$ consisting of all automorphisms which fix the vertices labelled $1, \ldots, 6$ in $\mathcal{F}_{1}$. Then, $x \widetilde{U} x^{-1}$ is the subgroup of $U$ consisting of all automorphisms fixing the vertices labelled $1^{\prime}, \ldots, 6^{\prime}$ in $\mathcal{F}_{2}$. Since $\widetilde{U}$ and $x \widetilde{U} x^{-1}$ are both subgroups of $U$, it follows that $U \cap x^{-1} U x \geq \widetilde{U}$. The reverse inclusion may be verified by checking cases for automorphisms not in $\widetilde{U}$. For example, if $u \in \operatorname{Aut}\left(\mathcal{T}_{2,2}\right)$ interchanges the two vertices on level 1 of the tree, then $x^{-1} u x$ maps the vertices of $\mathcal{F}_{1}$ labelled 5 and 6 , whose only common ancestor is the root, to vertices which have the vertex labelled $v$ as a common ancestor, and no such map is an automorphism of the tree. Hence,

$$
\left[x U x^{-1}: x U x^{-1} \cap U\right]=[U: \widetilde{U}]=32 .
$$

That this is not the minimum possible index will be seen by applying the tidying procedure to the subgroup U.

Step 1 It turns out that $U_{-1}=U \cap x^{-1} U x=\widetilde{U}$ is tidy above for $x$. To see this, Remark 1 tells us that it suffices to show that $\widetilde{U}_{1} \subseteq \widetilde{U}_{+} \widetilde{U}_{-1}$, where $\widetilde{U}_{1}=\widetilde{U} \cap x \widetilde{U} x^{-1}$ and $\widetilde{U}_{-1}=\widetilde{U} \cap x^{-1} \widetilde{U} x$. For this, observe that, since $\widetilde{U}$ is the fixator of $\mathcal{F}_{1}$ and $x \widetilde{U} x^{-1}$ is the fixator of $\mathcal{F}_{2}, \widetilde{U}_{1}$ is the fixator of $\mathcal{F}_{1} \cup \mathcal{F}_{2}$, see Figure 2. Furthermore, $\widetilde{U}_{-1}$ is the fixator of the tree $\mathcal{F}_{+}$shown in Figure 3 because $x$ maps the vertices $1^{\dagger}, \ldots, 5^{+}$to the vertices $1, \ldots, 5$; and $\widetilde{U}_{+}$is the fixator of the tree $\mathcal{V}_{+}$shown in Figure $4 .\left(\mathcal{V}_{+}\right.$includes the infinite path spanned by the images of the vertices 4 and 5 under positive powers of $x$. This is explained further in the next paragraph.) It follows that $\widetilde{U}_{+} \widetilde{U}_{-1}$ is the fixator of the tree $\mathcal{F}_{+} \cap \mathcal{V}_{+}$. This intersection is equal to $\mathcal{F}_{1}$ and so $\widetilde{U}_{+} \widetilde{U}_{-1}=\widetilde{U}$, which certainly contains $\widetilde{U}_{1}$. Therefore, $\widetilde{U}$ is tidy above. To be consistent with the notation of Section 4.1, $\widetilde{U}$ will now be denoted by $V$.

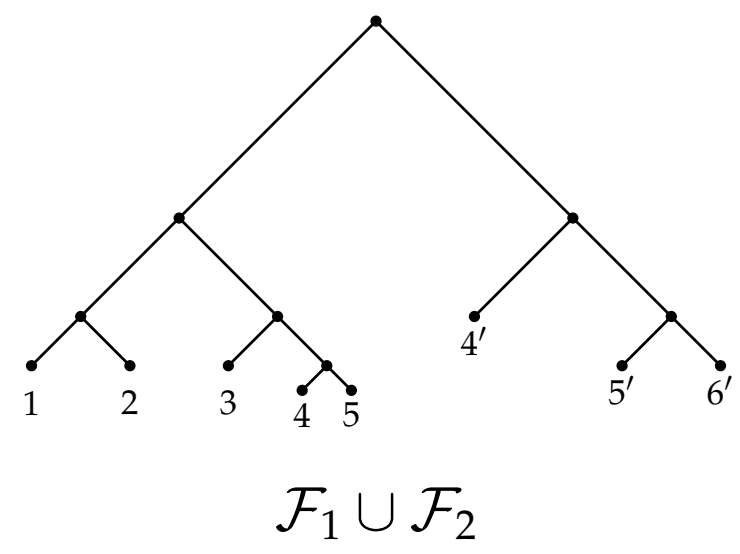

Figure 2. $\widetilde{U}_{1}$ is the fixator of the tree $\mathcal{F}_{1} \cup \mathcal{F}_{2}$. 


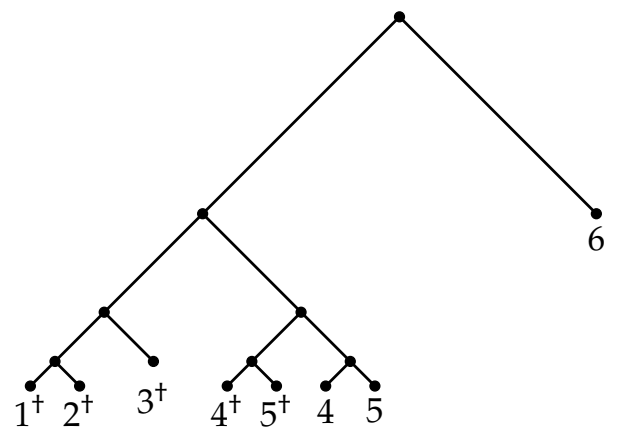

$\mathcal{F}_{+}$

Figure 3. $\widetilde{U}_{-1}$ is the fixator of the tree $\mathcal{F}_{+}$.

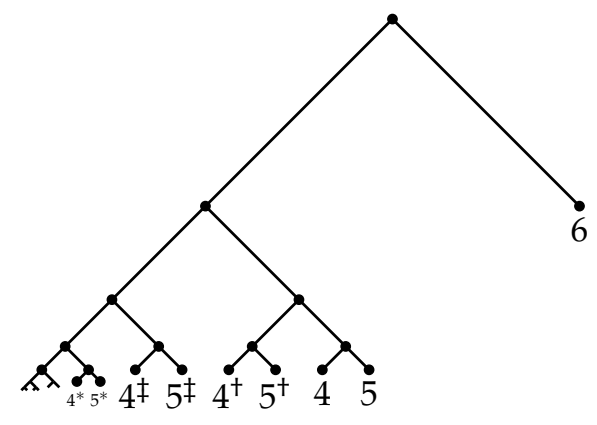

$\mathcal{V}_{-}$

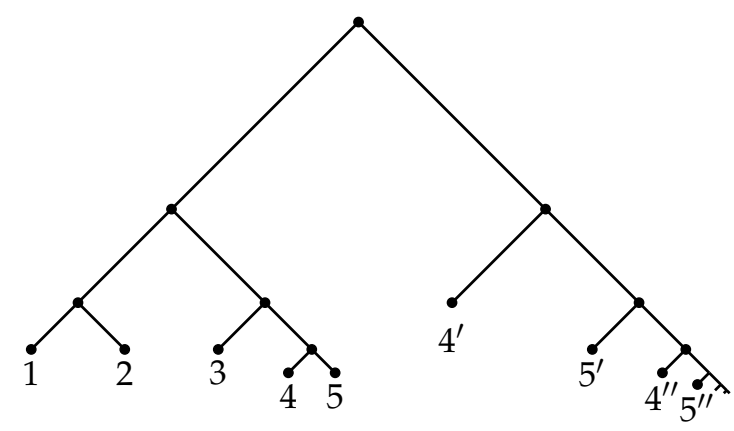

$\mathcal{V}_{+}$

Figure 4. Trees spanned by images of 4 and 5 under powers of $x$.

Steps 2 and 3 It further turns out that $\mathcal{L}_{V} \leq V$, so that $V$ (that is $\widetilde{U}$ ) is tidy below as well. To see this, it suffices to show that, if $v \in V_{+}$and $x^{N} v x^{-N} \in V_{-}$for some $N>0$, then $v \in V_{+} \cap V_{-}$.

By definition, $V_{+}=\bigcap_{n>0} x^{n} V x^{-n}$. As $x$ is iterated, the vertices 4 and 5 are pushed down two levels of $\mathcal{T}_{2,2}$ at a time and their images are at a distance 1 from a half-line descending from the vertex 6 , see Figure 4 . This half-line is part of an "axis" for $x$ that is translated down through distance 2 by $x$. Since $V$ fixes the vertices 4 and 5 , the given element $v \in V_{+}$fixes the tree $\mathcal{V}_{+}$shown in Figure 4.

By definition, $V_{-}=\bigcap_{n \leq 0} x^{n} V x^{-n}$. As $x$ is iterated, the vertices 4 and 5 are carried across the tree until they are the children $4^{\ddagger}$ and $5^{\ddagger}$ of vertex 2 , and then pushed down one level of $\mathcal{T}_{2,2}$ at a time, their images being at a distance 2 from a half-line descending from the vertex 1 , see Figure 4. This half-line is part of an "axis" for $x$ that is translated up through distance 1 by $x$. Since $V$ fixes the vertices 4 and $5, V_{-}$fixes the tree $\mathcal{V}_{-}$, shown in Figure 4, which includes this half-line and all vertices within distance 2 of it. For the particular element $v$, we have that $x^{N} v x^{-N} \in V_{-}$and so $v$ fixes all vertices in $\mathcal{V}_{-}$below level $N$. Since $v$ is a tree automorphism, it follows that $v$ fixes all vertices on the half-line descending from vertex 1 . However, it does not follow that $v$ fixes all images of vertices 4 and 5 above level $N$, and that must be shown in order to prove that $v \in V_{+} \cap V_{-}$. To show this, suppose for example that $v$ interchanges the vertices $4^{\dagger}$ and $5^{+}$. Then, $x v x^{-1}$ interchanges 4 and 5 , and so $x v x^{-1}$ is in $A u t\left(\mathcal{T}_{2,2}\right) \backslash V$. However, $x^{2} v x^{-2}$ interchanges $4^{\prime}$ and $5^{\prime}$ and so is not in $\operatorname{Aut}\left(\mathcal{T}_{2,2}\right)$. Similarly, $x^{n} v x^{-n}$ does not belong to $A u t\left(\mathcal{T}_{2,2}\right)$ for any $n \geq 2$, which contradicts that $x^{N} v x^{-N} \in V_{-}$. Hence, $v$ fixes the vertices $4^{\dagger}$ and $5^{\dagger}$. Similar arguments show that $v$ fixes all images of 4 and 5 in $\mathcal{V}_{-}$, and hence that $v \in V_{-}$, as claimed. 
Since every $v \in V_{+}$for which there is $N \geq 1$ with $x^{N_{v x}-N} \in V_{-}$must be in $V_{+} \cap V_{-}$, we have that $\mathcal{L}_{V} \leq V$ and hence that $V$ is tidy below for $x$. The scale of $x$ is therefore equal to

$$
\left[x V x^{-1}: x V x^{-1} \cap V\right]=\left[x \widetilde{U} x^{-1}: x \widetilde{U} x^{-1} \cap \widetilde{U}\right]=4 .
$$

Just as for automorphism groups of trees, the calculation of the scale of the non-uniscalar $x \in A A u t\left(\mathcal{T}_{2,2}\right)$ involves identifying an 'axis' for $x$. This axis consists of two half-lines with finite trees attached, one of which is translated through distance 2 and determines an attracting end for $x$, while the other is translated through distance 1 and determines a repelling end. A dynamical description of the action of an almost automorphism may be used in general for the calculation of the scale, although the dynamics can be more complicated as there may be several (and different numbers of) attracting and repelling ends and the almost automorphism may permute some of them. As seen here, the scale depends on more than just the speed with which the axis is translated towards or away from the ends, but also on the "thickness" of the axis. A similar description of the dynamics of the action of almost automorphisms is given in [36], which develops ideas in [37].

\section{Computing in t.d.l.c. Groups}

In the examples, computing the scale of the element or automorphism of $G$ requires a description of the element or automorphism, a description of a compact open subgroup, $U$ say, of $G$ and a method for calculating the images of $U$ under powers of the automorphism and forming their intersections. The different ways in which these things are done depends on the different concrete representations of $G$ in each case.

It is a truism that computation in a group depends on the description of the group. The computations may be at the level of an abstract group described by a presentation or, for some classes of groups, through a concrete representation. For example, finite groups have concrete representations as permutations (via Cayley's Theorem) and also as matrices (via the regular representation). Lie groups, too, have concrete representations as groups of isometries of symmetric spaces and also as groups of matrices (via the Lie algebra). The two types of concrete representation that exist in the cases of finite and Lie groups might be characterised as geometric and algebraic. They correspond to the two ways of thinking evident in synthetic and analytic geometry and perhaps too in analogue and digital computing.

Concrete descriptions of t.d.l.c. groups fit the pattern of being geometric or algebraic only to a more limited extent. The automorphism groups of trees in Example 2 are described in geometric terms and the scale is calculated in these terms as well, while the $p$-adic Lie groups in Example 4 are described and their scale calculated in algebraic terms. Some geometric and algebraic realisations of other t.d.l.c. groups, and the limitations of such represenations, are sketched in the next few paragraphs.

Many t.d.l.c. groups are defined geometrically as automorphism groups of buildings, see [15,38,39], and semisimple Lie groups over local fields. Kac-Moody groups over finite fields may also be represented as acting on buildings. Moreover, just as is the case for finite groups and Lie groups, every compactly generated t.d.l.c. group has a geometric representation via an action on a Cayley-Abels graph, $[40,41]$. Cayley-Abels graphs are unique only up to quasi-isometry however and, although they can be used to derive bounds on integer invariants of t.d.l.c. groups, see ([30] Proposition 4.6) and [42,43], they do not provide an effective method for performing precise calculations of invariants such as the scale unless the graph structure is understood in as much detail as it is for buildings. The limitation of the Cayley-Abels geometric representation therefore is that, unlike the cases of geometries for finite and Lie groups, it is not well understood for general t.d.l.c. groups: no such graph has been described for Neretin's group for example.

The limitation of algebraic realisations (strictly conceived) is that t.d.l.c. groups such as $\operatorname{Aut}\left(\mathcal{T}_{q+1}\right)$ and Neretin's group do not have finite-dimensional linear representations. However, there is a possible substitute for algebraic realisations of these groups. The calculation of the scale on Neretin's group illustrated in Example 5 uses an approach that is not readily characterised as geometric or algebraic. 
Although the axes of translation featuring in the dynamical description of the almost automorphism are geometric, the axes and translation distance alone do not determine the scale. The full information required is encoded in the pair of finite trees representing the almost automorphism. This information is combinatorial in nature and says how the almost automorphism commensurates the totally disconnected compact group $\operatorname{Aut}\left(\mathcal{T}_{2,2}\right)$. Since abstract t.d.l.c. groups always contain a compact open, and hence commensurated, subgroup, they may be realised concretely as groups of commensurators quite generally, see $[29,30,35]$. Moreover, given any pair $(G, H)$ such that $H$ is a commensurated subgroup of $G$, a t.d.l.c. group $\widetilde{G}$, called the relative profinite completion, may be defined in which the closure of $H$ is a compact open subgroup of $\widetilde{G}$, see [44] and the references therein. Examples of such pairs include the group $\operatorname{PSL}(n, \mathbb{Z}[1 / p])$ and its commenustared subgroup $\operatorname{PSL}(n, \mathbb{Z})$, in which case the relative profinite completion is isomorphic to $\operatorname{PSL}\left(n, \mathbb{Q}_{p}\right)$; and the Baumslag-Solitar group $B S(m, n):=\left\langle a, t: t a^{m} t^{-1}=a^{n}\right\rangle$ and commensurated subgroup $\langle a\rangle$, in which case the relative profinite completion is described in [45].

These examples suggest that a suitable substitute for the adjoint representation might be to represent t.d.l.c. groups concretely as commensurators. This idea is lent support by the fact that, in $p$-adic Lie groups, locally normal subgroups (in the local structure theory developed in [46]) correspond to ideals in the Lie algebra, and that the scale (which is defined in terms of commensuration) may be expressed in terms of eigenvalues. Representing general t.d.l.c. groups as commensurator groups poses challenges comparable with those facing the use of Cayley-Abels graphs however.

A practical test of any description of a t.d.l.c. group is whether it facilitates calculation of the scale and identification of tidy subgroups. Seeking descriptions which pass that test for more groups is a task for further investigations. These investigations might guide a possible"classification" of topologically simple t.d.l.c. groups in which the groups are arranged into types according to their best method of concrete description. While linear representations largely suffice in the cases of Lie and finite groups, that will not be the case for t.d.l.c. groups because automorphism groups of trees, Neretin's group and most Kac-Moody groups are not linear, and there may be many others yet to be discovered. Geometric, linear and commensurator descriptions may all be required but it is not clear that they will suffice.

S. Smith has shown, see [47], how uncountably many simple discrete groups may be used to produce uncountably many topologically simple, compactly generated t.d.l.c. groups. Smith's groups act on trees (not locally finite ones though) and so have a geometric description, modulo an infinite discrete group, which facilitates calculation of the scale. There seems to be no reason that there might not be other ways of constructing uncountable families of simple t.d.l.c. groups however. These, presumably, would also be described modulo objects in some class known to be uncountable. Arranging groups constructed in these ad hoc ways according to their method of concrete description, and their local structure as defined in [46], may be the best that can be hoped for in the direction of a classification.

Calculating the scale of an element of a t.d.l.c. group can use only a finite amount of information. To calculate the scale, at least in principle, of any element in any compactly generated, topologically simple t.d.l.c. group would, since there are uncountably many of them, entail describing the group up to some finite approximation, or modulo information not relevant to the calculation, and then identifying the element in it up to a finite approximation. It would ultimately be desirable to implement this calculation in computer software. Indeed, it might be argued that such implementation would be the benchmark of success for a theory of t.d.l.c. groups and a categorising, or sorting into types, of the simple ones.

Acknowledgments: The support of the ARC Discovery Grant DP150100060 is gratefully acknowledged.

Conflicts of Interest: The author declares no conflict of interest. 


\section{References}

1. Van Dantzig, D. Zur topologischen Algebra III: Brouwersche und Cantorsche Gruppen. Compos. Math. 1936, 3, 408-426.

2. Hewitt, E.; Ross, K.A. Abstract Harmonic Analysis I; Grundlehren der mathematischen Wissenschaften, Bd 115; Springer: Berlin/Göttingen/Heidelberg, Germany, 1963.

3. Möller, R.G. Structure theory of totally disconnected locally compact groups via graphs and permutations. Can. J. Math. 2002, 54, 795-827.

4. Willis, G.A. The structure of totally disconnected, locally compact groups. Math. Ann. 1994, 300, 341-363.

5. Willis, G.A. Further properties of the scale function on totally disconnected groups. J. Algebra 2001, 237, 142-164.

6. Willis, G.A. Tidy subgroups for commuting automorphisms of totally disconnected groups: An analogue of simultaneous triangularisation of matrices. N. Y. J. Math. 2004, 10, 1-35.

7. Jaworski, W.; Rosenblatt, J.M.; Willis, G.A. Concentration functions in locally compact groups. Math. Ann. 1996, 305, 673-691.

8. Previts, W.H.; Wu, T.-S. On tidy subgroups of locally compact totally disconnected groups. Bull. Aust. Math. Soc. 2002, 65, 485-490.

9. Willis, G.A. The scale and tidy subgroups for endomorphisms of totally disconnected locally compact groups. Math. Ann. 2015, 361, 403-442.

10. Willis, G.A. Totally disconnected groups and proofs of conjectures of Hofmann and Mukherjea. Bull. Aust. Math. Soc. 1995, 51, 489-494.

11. Caprace, P.-E.; Reid, C.D.; Willis, G.A. Limits of Contraction Groups and the Tits Core. J. Lie Theory 2014, 24, 957-967.

12. Hofmann, K.H.; Willis, G.A. Continuity characterizing totally disconnected locally compact groups. J. Lie Theory 2015, 25, 1-7.

13. Braconnier, J. Groupes d'automorphismes d'un groupe localement compact. Comptes Rendus Acad. Sci. Paris 1945, 220, 382-384.

14. Willis, G.A. Conjugation weights and weighted convolution algebras on totally disconnected, locally compact groups. In Proceedings of the Centre for Mathematics and Its Applications, Proceedings of the AMSI Conference on Harmonic Analysis and Applications, Macquarie University, Sydney, Australia, 7-11 February 2011; Duong, X., Hogan, J., Meaney, C., Sikora, A., Eds.; The Australian National University, Mathematical Sciences Institute, Centre for Mathematics \& its Applications: Canberra, Australian, 2012; Volume 45, pp. 136-147.

15. Tits, J. Sur le groupe des automorphismes d'un arbre. In Essays on Topology and Related Topics (Mémoires Dédiés à Georges de Rham); Springer: New York, NY, USA, 1970; pp. 188-211.

16. Abramenko, P.; Brown, K.S. Buildings: Theory and Application; Graduate Texts in Mathematics; Springer: New York, NY, USA, 2008; Volume 248.

17. Glöckner, H. Scale functions on p-adic Lie groups. Manuscr. Math. 1998, 97, 205-215.

18. Wang, J.S.P. The Mautner phenomenon for p-adic Lie groups. Math. Z. 1984, 185, 403-412.

19. Baumgartner, U.; Willis, G.A. Contraction groups for automorphisms of totally disconnected groups. Isr. J. Math. 2004, 142, 221-248.

20. Glöckner, H. Scale Functions on Linear Groups Over Local Skew Fields. J. Algebra 1998, 205, 525-541.

21. Glöckner, H.; Willis, G.A. Uniscalar p-adic Lie groups. Forum Math. 2001, 13, 413-421.

22. Parreau, A. Sous-groupes elliptiques de groupes linéaires sur un corps valué. (French) [Elliptic subgroups of linear groups over a field with valuation]. J. Lie Theory 2003 13, 271-278.

23. Kepert, A.; Willis, G.A. Scale functions and tree ends. J. Aust. Math. Soc. 2001, 70, $273-292$.

24. Willis, G.A. Compact open subgroups in simple totally disconnected groups. J. Algebra 2007, 312, 405-417.

25. Neretin, Y.A. Combinatorial analogues of the group of diffeomorphisms of the circle. (Russian. Russian summary). Izv. Ross. Akad. Nauk Ser. Mat. 1992, 56, 1072-1085; translation in Russ. Acad. Sci. Izv. Math. 1993, 41, 337-349.

26. Neretin, Yu.A. Groups of hierarchomorphisms of trees and related hilbert spaces. J. Funct. Anal. 2003, 200, 505-535.

27. Kapoudjian, C. Simplicity of Neretin's group of spheromorphisms. Ann. Inst. Fourier (Grenoble) 1999, 49, 1225-1240. 
28. Bader, U.; Caprace, P.-E.; Gelander, T.; Mozes, S. Simple groups without lattices. Bull. Lond. Math. Soc. 2012, 44, 55-67.

29. Barnea, Y.; Ershov, M.; Weigel, T. Abstract commensurators of profinite groups. Trans. Am. Math. Soc. 2011, 363, 5381-5417.

30. Caprace, P.-E.; De Medts, T. Simple locally compact groups acting on trees and their germs of automorphisms. Transform. Groups 2011, 16, 375-411.

31. Le Boudec, A. Groups acting on trees with almost prescribed local action. Comment. Math. Helv. 2016, 91, 253-293.

32. Burger, M.; Mozes, S. Groups acting on trees: From local to global structure. Inst. Hautes Études Sci. Publ. Math. 2000, 92, 113-150.

33. Cannon, J.W.; Floyd, W.J.; Parry, W.R. Introductory notes on Richard Thompson's groups. Enseign. Math. 1996, 42, 215-256.

34. Higman, G. Finitely presented infinite simple groups. In Notes on Pure Mathematics 8; Australian National University: Canberra, Australia, 1974.

35. Röver, C. Abstract commensurators of groups acting on rooted trees. Geom. Dedicata 2002, 94, 45-61.

36. Salazar-Díaz, O. P. Thompson's group $V$ from a dynamical viewpoint. Int. J. Algebra Comput. 2010, $20,39-70$.

37. Brin, M. Higher dimensional Thompson's groups. Geom. Dedicata 2004, 108, 163-192.

38. Haglund, F.; Paulin, F. Simplicité de groupes d'automorphismes d'espaces à courbure négative. Geom. Topol. Monogr. 1998, 1, 181-248.

39. Nebbia, C. Minimally almost periodic totally disconnected groups. Proc. Am. Math. Soc. 1999, 128, $347-351$.

40. Abels, H. Specker-Kompaktifizierungen von lokal kompakten topologischen Gruppen. Math. Z. 1974, 138, 325-361.

41. Monod, N. Continuous Bounded Cohomology of Locally Compact Groups; Lecture Notes in Mathematics; Springer: Berlin, Germany, 2001; Volume 1758.

42. Baumgartner, U.; Möller, R.; Willis, G.A. Hyperbolic groups have flat-rank at most 1. Isr. J. Math. 2012, 190, 365-388.

43. Reid, C.D.; Wesolek, P.R. The essentially chief series of a compactly generated locally compact group. Math. Ann. 2017, doi:10.1007/s00208-017-1597-0.

44. Reid, C.D.; Wesolek, P.R. Homomorphisms in totally disconnected, locally compact groups with dense image. arXiv 2015, doi:arXiv:1509.00156v1.

45. Elder, M.; Willis, G.A. Totally disconnected groups from Baumslag-Solitar groups. arXiv 2013, arXiv:1301.4775.

46. Caprace, P.-E.; Reid, C.D.; Willis, G.A. Locally normal subgroups of totally disconnected groups. Part II: Compactly generated simple groups. Forum Math. Sigma 2017, 5, doi:10.1017/fms.2017.9.

47. Smith, S.M. A product for permutation groups and topological groups. Duke Math. J. 2017, 166, $2965-2999$. 